\title{
Preparation and Characterisation of Nobiletin-Loaded Nanostructured Lipid Carriers
}

\author{
Wei Huang, ${ }^{1,2}$ Huating Dou, ${ }^{1}$ Houjiu Wu, ${ }^{1}$ Zhigao Sun, ${ }^{1}$ Hua Wang, ${ }^{1}$ and Linhua Huang ${ }^{1}$ \\ ${ }^{1}$ Citrus Research Institute, Southwest University and Chinese Academy of Agricultural Sciences, Chongqing 400715, China \\ ${ }^{2}$ College of Forestry and Life Science, Chongqing University of Arts and Sciences, Chongqing 402160, China \\ Correspondence should be addressed to Huating Dou; hdou33880@yahoo.com
}

Received 24 February 2017; Revised 6 July 2017; Accepted 19 July 2017; Published 17 August 2017

Academic Editor: Bo Tan

Copyright (c) 2017 Wei Huang et al. This is an open access article distributed under the Creative Commons Attribution License, which permits unrestricted use, distribution, and reproduction in any medium, provided the original work is properly cited.

\begin{abstract}
The objective of this manuscript was to investigate and optimise the potential of nanostructured lipid carriers (NLCs) as a carrier system for nobiletin (NOB), which was prepared by high-pressure homogenisation method. Additionally, this study was focused on the application of NOB-loaded NLC (NOB-NLC) in functional food. Response surface method with a three-level Box-Behnken design was validated through analysis of variance, and the robustness of the design was confirmed through the correspondence between the values measured in the experiments and the predicted ones. Properties of the prepared NOB-NLC, such as $Z$ average, polydispersity, entrapment efficiency, zeta potential, morphology, and crystallinity, were investigated. NOB-NLC exhibited a spherical shape with a diameter of $112.27 \pm 5.33 \mathrm{~nm}$, zeta potential of $-35.1 \pm 2.94 \mathrm{mV}$, a polydispersity index of $0.251 \pm 0.058$, and an EE of $81.06 \% \pm 6.02 \%$. Results from X-ray diffraction and differential scanning calorimetry of NOB-NLC reviewed that the NOB crystal might be converted to an amorphous state. Fourier transform infrared spectroscopic analysis demonstrated that chemical interaction was absent between the compound and lipid mixture in NOB-NLC.
\end{abstract}

\section{Introduction}

Nobiletin $\left(5,6,7,8,3^{\prime}, 4^{\prime}\right.$-hexametoxyflavone, NOB) is a citrus polymethoxylated flavone that possesses an extensive range of health benefits among others, including antioxidant activity, glucose regulation, and fat metabolism [1]. It was previously reported that nobiletin could inhibit tumor angiogenesis by regulating Src, FAK, and STAT3 signalling through PXN in ER+ breast cancer cells [2] and also showed its ameliorative effects toward ischemia-reperfusion injury by suppressing the function of Kupffer cells after liver transplantation in rats [3]. Furthermore, nobiletin has been found to have positive effects of nobiletin on propofol-mediated antiinflammatory as well as having neuroprotective effect [4]. It may represent beneficial drug candidates for the treatment and prevention of Alzheimer's and Parkinson's disease because of its naturally occurring phytochemicals [5]. Similar to other flavonoids, NOB exists widely in nature and is found mainly in various citrus peels. Therefore, a citrus processing waste can be potentially converted into a valuable functional ingredient. However, NOB exhibits poor water solubility because all hydroxyl groups in the structure are methoxylated, leading to low oral bioavailability, thus limiting the practical uses of NOB [6].

Delivery systems using lipid-based bioactive components as promising carriers are getting increasing attention nowadays because of their potential to increase solubility and improve bioavailability of water-insoluble and/or lipophilic bioactive components [7]. NLCs are second-generation lipid nanoparticles distinguished from SLN as described by Müller et al. $[8,9]$. NLCs possess the advantages related to SLNs, such as feasibility for encapsulation of hydrophobic and hydrophilic compounds and the capability to impart controlled and sustained release characteristics over free-state compound. Furthermore, NLCs have the ability to protect compounds from chemical, physical, oxidative, or light degradation; are of extremely low toxicity; and are also of low cost $[10,11]$. NLCs are derived from oil-water emulsions and SLNs by preparing a liquid lipid through a matrix of a solid lipid. Compared with SLNs, this matrix has a less-ordered crystalline nanostructure 
that imparts higher loading capacity for the compound, overcomes the drawback of the compound expulsion from the lipid core, and now is popularly studied and used in pharmaceutical, cosmetic, and food products $[12,13]$. In spite of this, there has been no report about the application of NLC on delivery of NOB. Quite a few examinations have been undertaken to improve the healthful potential of NOB for oral administration by choosing appropriate delivery system, including chitosan-based microemulsions loaded with NOB for intravenous injection [14], self-nanoemulsifying delivery systems [15], NOB-loaded chitosan nanoparticles [16], self-assembling NOB proliposomes [17], and NOB-loaded solid lipid nanoparticles (SLNs) [18]. According to recent researches, nanoemulsion-filled hydrogel could achieve the sustained release and absorption of nobiletin and prevent its precipitation in the gastrointestinal tract [19]. Taken together, these previous findings provided the foundation and evidence for further research on NOB-loaded NLC.

The objectives of this research were as follows: firstly, to study the suitability and the effectiveness of NLCs as a new type carrier system to encapsulate NOB and, secondly, to investigate the characteristics of the prepared NLC. To perform and optimise the formulations, a Box-Behnken design (BBD) of three factors and three levels was used, and highpressure homogenisation technology was chosen by considering industrial needs $[12,20]$. Furthermore, the following characteristics of NLC system were fully examined: particle size, polydispersity index (PDI), zeta potential, entrapment efficiency (EE), morphology and crystallinity through dynamic light scattering (DLS), high-performance liquid chromatography method (HPLC), transition electron microscopy (TEM), differential scanning calorimetry (DSC), Fourier transform infrared (FT-IR), and X-ray diffraction (XRD).

\section{Materials and Methods}

2.1. Materials. NOB was acquired from Lisi Bio-Tech Inc. (Xi'an, China). Labrafac lipophile WL 1349 and Geleol monoand biglycerides NF were obtained from Gattefosse (SaintPriest Cedex, France). Lecithin (containing 90\% soybean phosphatidylcholine) was obtained from Shanghai Taiwei Pharmaceutical Co. Ltd. (Shanghai, China). Poloxamer 188 (Pluronic $\mathrm{F} 68^{\circledR}$ ) was a generous gift from BASF (Ludwigshafen, Germany). Methanol and acetonitrile provided by Sigma Co. (St. Louis, MO, USA) were of HPLC grade. Ultrapure water was prepared through a Direct- $\mathrm{Q}^{\mathrm{TM}}$ instrument (Millipore, Molsheim, France).

2.2. Preparation of NOB-NLC. NOB-NLC was prepared through melt-emulsification technique and high-pressure homogenisation [21]. Briefly, $3 \mathrm{~mL}$ ethanol was added as cosolvent to the lipid phase, which was evenly composed of NOB, solid lipid, liquid lipid, and lecithin. A total of $100 \mathrm{~mL}$ each of aqueous phase, including surfactant, and lipid phase was heated to $75 \pm 3^{\circ} \mathrm{C}$ in a water bath. Subsequently, the lipid matrix was injected into the aqueous matrix, and the mixture phase was stirred at $500 \mathrm{rpm}$ for $10 \mathrm{~min}$ and then mixed by a Lab high-shear homogeniser SRH-S300 (Siehe Industry, Shanghai, China) at $10000 \mathrm{rpm}$ for $2 \mathrm{~min}$ at
TABLE 1: Variables with respective coded levels of the Box-Behnken design for the preparation and optimization of NOB-NLC.

\begin{tabular}{lccc}
\hline Variables & \multicolumn{3}{c}{ Levels } \\
\hline Independent variables & $\begin{array}{c}\text { Low } \\
(-1)\end{array}$ & $\begin{array}{c}\text { Medium } \\
(0)\end{array}$ & $\begin{array}{c}\text { High } \\
(+1)\end{array}$ \\
\hline$X_{1}:$ emulsifier-to-lipid ratio & 1 & 1.75 & 2.5 \\
$X_{2}:$ lecithin-to-Poloxamer 188 ratio & 0.5 & 1.25 & 2 \\
$X_{3}:$ liquid lipid-to-solid liquid ratio & 0.33 & 1 & 1.67 \\
\hline Dependent variables & \multicolumn{3}{c}{ Constraints } \\
\hline$Y_{1}:$ mean particle size $(\mathrm{nm})$ & \multicolumn{3}{c}{ Minimum } \\
$Y_{2}:$ encapsulation efficiency $(\%)$ & \multicolumn{3}{c}{ Maximum } \\
\hline
\end{tabular}

the same temperature. Afterwards, the coarse emulsion was homogenised using a nanohomogeniser machine AH100D (ATS Engineering Inc., Vancouver, Canada) under 800 bar for 10 cycles. Finally, the resulting nanoemulsion was allowed to cool down to room temperature to form NOB-NLC.

2.3. Box-Behnken Design for Optimisation of NOB-NLC. A response surface method (RSM) based on BBD was employed, and a 17-run design with three factors and three levels, which included 12 factorial points and 5 axial points, was carried out in the present work [22]. Meanwhile, Design Expert $^{\circledR}$ 8.0.5 software (Stat-Ease Inc., Minneapolis, USA) was applied to determine the response patterns and then to establish models, which are shown below:

$$
\begin{aligned}
Y= & \lambda_{0}+\lambda_{1} X_{1}+\lambda_{2} X_{2}+\lambda_{3} X_{3}+\lambda_{12} X_{1} X_{2} \\
& +\lambda_{13} X_{1} X_{3}+\lambda_{23} X_{2} X_{3}+\lambda_{11} X_{1}^{2}+\lambda_{22} X_{2}^{2} \\
& +\lambda_{33} X_{3}^{2} .
\end{aligned}
$$

As shown in (1), the measured responses $(Y)$ were mean particle size $\left(Y_{1}\right)$ and $\mathrm{EE}\left(Y_{2}\right)$. The three independent variables selected were emulsifier-to-lipid ratio $\left(X_{1}\right)$, lecithin-toPoloxamer 188 ratio $\left(X_{2}\right)$, and liquid lipid-to-solid lipid ratio $\left(X_{3}\right)$ [23]. For each factor, high, medium, and low levels were represented by codes 1,0 , and -1 , respectively (Table 1 ). $\lambda_{0}$ is the intercept; $\lambda_{1}, \lambda_{2}$, and $\lambda_{3}$ are the linear coefficients; $\lambda_{12}$, $\lambda_{13}$, and $\lambda_{23}$ are the interaction coefficients; and $\lambda_{11}, \lambda_{22}$, and $\lambda_{33}$ are the quadratic coefficients.

2.4. Z-Average, PDI, and Zeta Potential Characterisation. Zaverage, PDI, and zeta potential of NOB-NLC were analysed through DLS using a Zeta Analyser ZEN-3600 (Malvern Instruments, Worcester, UK). Sample measurements were taken in triplicate at $25^{\circ} \mathrm{C}$ with a fixed scattering angle of $173^{\circ}$. The collected data were then analysed through the manufacturer-supplied software from Malvern Instruments [24].

2.5. Entrapment Efficiency and High-Performance Liquid Chromatography Measurement. The EE of NOB-NLC was determined by measuring free NOB in the aqueous phase, which was separated using ultrafiltration centrifugation [21, 25]. A total of $500 \mu \mathrm{L}$ NOB-NLC was added to the upper 
chamber of Centrifugal Filter Unit $100 \mathrm{kDa}$ (Millipore Co., Boston, USA) and then centrifuged at $3000 \mathrm{rpm}$ for $20 \mathrm{~min}$ at $4^{\circ} \mathrm{C}$ through UniCen MR (Herolab, Germany) [26]. The filtrate was dissolved in methanol and analysed through HPLC using Dionex UltiMate 3000 (Sunnyvale, USA) with C18 column. Chromatographic analysis was performed at $30^{\circ} \mathrm{C}$ and $333 \mathrm{~nm}$ using ultrapure water and acetonitrile $(55: 45 \mathrm{v} / \mathrm{v})$ as the mobile phase, with injection volume of $20 \mu \mathrm{L}$ and a flow rate of $1.0 \mathrm{~mL} / \mathrm{min}$. Acetonitrile and ultrapure water were previously eluted through a $0.22 \mu \mathrm{m}$ membrane filter. The EE was calculated through the following equation [27]:

$$
\mathrm{EE}(\%)=\frac{W_{\text {total }}-W_{\text {free }}}{W_{\text {total }}} \times 100,
$$

where $W_{\text {total }}$ is the mass of initial NOB used for the assay and $W_{\text {free }}$ is the mass of free NOB in the aqueous phase.

2.6. Transition Electron Microscopy Analysis. The morphological characteristics of NOB-NLC were observed using TEM JEM-2100 (JEOL Ltd., Tokyo, Japan) at $200 \mathrm{kV}$. The samples were dropped onto Formvar/Carbon 230 Mesh copper grids (Zhongjingkeyi Technology Co. Ltd., Beijing, China), air-dried at room temperature of about $25 \pm 3^{\circ} \mathrm{C}$ for $24 \mathrm{~h}$, and negatively stained with phosphotungstic acid (1\% w/v) for approximately 20 min before observation [26].

2.7. Differential Scanning Calorimetry Measurement. The thermograms of freeze-dried NOB-NLC, blank NLC, and NOB were analysed using DSC Q20 (TA Instruments, New Castle, USA). $3 \mathrm{mg}$ of the samples was placed into standard aluminum pans, and the temperature was then increased from $25^{\circ} \mathrm{C}$ to $240^{\circ} \mathrm{C}$ at a heating or cooling scanning rate of $10^{\circ} \mathrm{C} \mathrm{min}^{-1}$. During thermal scans, dry nitrogen was used as effluent gas and an empty pan as a reference for the analysis $[25,26,28]$.

2.8. Fourier Transform Infrared Spectroscopy. The FT-IR spectrum of freeze-dried NOB-NLC, blank NLC, and NOB was examined using FT-IR spectrometer Nicolet 6700 (Madison, USA). The samples were scanned over a wavenumber [29], ranging from $550 \mathrm{~cm}^{-1}$ to $4000 \mathrm{~cm}^{-1}$.

2.9. X-Ray Diffraction Measurements. X-ray diffractometer $X^{\prime}$ Pert ${ }^{3}$ Power (PANalytical B.V., Almelo, The Netherlands) was chosen to collect and compare the XRD intensity data of lyophilised NOB-NLC, lyophilised blank NLC, and NOB. $\mathrm{XRD}$ analysis was performed under $\mathrm{CuK} \alpha$ radiation $(\lambda=$ $1.54056 \AA$, voltage $40 \mathrm{kV}$, and current $40 \mathrm{~mA}$ ) across the diffraction angle range of $0^{\circ} \leq 2 \theta \leq 90^{\circ}$ (step size: $0.013^{\circ}$; scan step time: 8.67 s) [30].

2.10. Data Analysis. All of the experiments were run in triplicate, and results were presented as mean \pm SD. Design Expert 8.0.5 software was employed for design and analysis of the data for RSM. Differences were regarded as significant when $P$ value $<0.05$.

\section{Results and Discussion}

3.1. Formulation Optimisation of NOB-NLC. Based on the $\mathrm{BBD}$ design, 17 different formulations were carried out to determine the most critical independent variables, and the responses are summarised in Table 2. The final parameters that most intensely affected the properties of NOB-NLC were identified to be emulsifier-to-lipid ratio $\left(X_{1}\right)$, lecithin-toPoloxamer 188 ratio $\left(X_{2}\right)$, and liquid lipid-to-solid lipid ratio $\left(X_{3}\right)$. The polynomial equations were statistically validated through analysis of variance, and a summary of the results of regression analysis of responses is demonstrated in Table 3. The $3 \mathrm{D}$ graphs of the response surface plotted to represent the effects of the independent factors on measured responses of mean particle size and EE are shown in Figures 1 and 2.

3.1.1. Effect on Mean Particle Size. The mean particle size $\left(Y_{1}\right)$ varied from $95.7 \mathrm{~nm}$ (Formulation (13)) to $186.325 \mathrm{~nm}$ (Formulation (2)), whereas the average was $139.96 \mathrm{~nm}$ as shown in Table 2. The effect of independent variables on mean particle size has been presented through the quadratic equation as follows:

$$
\begin{aligned}
Y_{1}= & 332.35-95.38 X_{1}-93.43 X_{2}-105.30 X_{3} \\
& -30.67 X_{1} X_{2}-24.53 X_{1} X_{3}-2.42 X_{2} X_{3} \\
& +41.59 X_{1}^{2}+55.32 X_{2}^{2}+57.97 X_{3}^{2} .
\end{aligned}
$$

Equation (3) revealed negative effects on the variables $X_{1}$, $X_{2}$, and $X_{3}$ on $Y_{1}$, as indicated by the negative value before the variable in the quadratic equation. Higher emulsifier-tolipid ratio may promote formation of smaller particles and provide higher stabilisation of nanosystem because of the reduction in interfacial tension between the lipid matrix and the hydrophilic phase [31]. The results showed that as liquid lipid-to-solid lipid ratio increased, particle size decreased from $175.39 \mathrm{~nm}$ (Formulation (6)) to $96.93 \mathrm{~nm}$ (Formulation (8)), which can be attributed to the increased amount of solid lipids that tend to facilitate the aggregation of particles.

The 3D surface plots for the effect of interaction between $X_{1}-X_{2}, X_{1}-X_{3}$, and $X_{2}-X_{3}$ on particle size are shown in Figures $1(\mathrm{a})-1$ (c). As shown in Table 3 , all independent factors have significant effect on the mean particle size of NOBNLC $(P<0.05)$, particularly the liquid lipid-to-solid lipid ratio $\left(X_{3}\right.$ and $\left.X_{3}{ }^{2}\right)$. Furthermore, the interaction term of emulsifier-to-lipid ratio and lecithin-to-Poloxamer 188 ratio surfactant $\left(X_{1} X_{2}\right)$ is also significant $(P<0.05)$, which is consistent with the analysis $3 \mathrm{D}$ plot of response surface (Figure 1(a)). In addition, the correlation coefficient $\left(R^{2}\right)$ was 0.9427 , indicating that merely $5.73 \%$ of total variables could not be illustrated by the model. The coefficient of variation was at $8.9 \%$, and the low value suggested that the observed values were consistent with the predicted ones. The signal-to-noise ratio of the model was evaluated by "Adeq Precision" [32], with the value of 9.208 ( $>4)$, which indicated the sufficiency of model with mean particle size [33].

3.1.2. Effect on Entrapment Efficiency. In the case of EE, the value ranged from $59.35 \%$ (Formulation (8)) to $88.72 \%$ 
TABLE 2: Observed response in Box-Behnken design for preparation and optimization of NOB-NLC formulations with predicted values generated by Design Expert software.

\begin{tabular}{|c|c|c|c|c|c|c|c|}
\hline \multirow{3}{*}{ Formulations } & \multicolumn{3}{|c|}{ Independent variables } & \multicolumn{4}{|c|}{ Dependent variables } \\
\hline & \multirow{2}{*}{$X_{1}$} & \multirow{2}{*}{$X_{2}$} & \multirow{2}{*}{$X_{3}$} & \multicolumn{2}{|c|}{ Observed value } & \multicolumn{2}{|c|}{ Predicted value } \\
\hline & & & & $Y_{1}$ & $Y_{2}$ & $Y_{1}$ & $Y_{2}$ \\
\hline (1) & 1.00 & 0.50 & 1.00 & $159.27 \pm 9.52$ & $74.45 \pm 3.01$ & 157.26 & 71.42 \\
\hline (2) & 2.50 & 0.50 & 1.00 & $186.32 \pm 6.20$ & $85.73 \pm 6.36$ & 172.72 & 83.38 \\
\hline (3) & 1.00 & 2.00 & 1.00 & $161.32 \pm 10.35$ & $84.89 \pm 5.07$ & 174.92 & 87.24 \\
\hline (4) & 2.50 & 2.00 & 1.00 & $119.38 \pm 6.60$ & $65.14 \pm 3.81$ & 121.38 & 68.17 \\
\hline (5) & 1.00 & 1.25 & 0.33 & $181.37 \pm 9.05$ & $80.31 \pm 4.92$ & 172.34 & 81.30 \\
\hline (6) & 2.50 & 1.25 & 0.33 & $175.39 \pm 10.40$ & $75.46 \pm 3.75$ & 177.96 & 75.77 \\
\hline (7) & 1.00 & 1.25 & 1.67 & $152.22 \pm 7.73$ & $60.25 \pm 3.77$ & 149.65 & 59.94 \\
\hline (8) & 2.50 & 1.25 & 1.67 & $96.93 \pm 5.61$ & $59.35 \pm 5.54$ & 105.95 & 58.36 \\
\hline (9) & 1.75 & 0.50 & 0.33 & $179.04 \pm 9.90$ & $80.58 \pm 4.06$ & 190.08 & 82.62 \\
\hline (10) & 1.75 & 2.00 & 0.33 & $180.25 \pm 8.06$ & $86.71 \pm 6.50$ & 175.67 & 83.38 \\
\hline (11) & 1.75 & 0.50 & 1.67 & $140.59 \pm 6.49$ & $60.36 \pm 4.22$ & 145.17 & 63.69 \\
\hline (12) & 1.75 & 2.00 & 1.67 & $136.92 \pm 5.38$ & $65.58 \pm 4.75$ & 125.89 & 63.54 \\
\hline (13) & 1.75 & 1.25 & 1.00 & $95.70 \pm 7.69$ & $79.65 \pm 5.90$ & 102.06 & 83.67 \\
\hline (14) & 1.75 & 1.25 & 1.00 & $96.13 \pm 7.70$ & $85.24 \pm 6.07$ & 102.06 & 83.67 \\
\hline (15) & 1.75 & 1.25 & 1.00 & $102.37 \pm 6.54$ & $83.79 \pm 6.33$ & 102.06 & 83.67 \\
\hline (16) & 1.75 & 1.25 & 1.00 & $115.14 \pm 8.25$ & $80.93 \pm 4.61$ & 102.06 & 83.67 \\
\hline$(17)$ & 1.75 & 1.25 & 1.00 & $100.98 \pm 7.33$ & $88.72 \pm 3.95$ & 102.06 & 83.67 \\
\hline
\end{tabular}

$X_{1}=$ emulsifier-to-lipid ratio, $X_{2}=$ lecithin-to-Poloxamer 188 ratio, $X_{3}=$ liquid lipid-to-solid liquid ratio, $Y_{1}=$ mean particle size $(\mathrm{nm})$, and $Y_{2}=$ encapsulation efficiency (\%).

TABLE 3: Statistical analysis results of lack of fit test and model summary for mean particle size and entrapment efficiency.

\begin{tabular}{|c|c|c|c|c|c|c|c|c|}
\hline \multirow{2}{*}{ Source } & \multicolumn{4}{|c|}{$Y_{1}$} & \multicolumn{4}{|c|}{$Y_{2}$} \\
\hline & Sum of squares & Mean square & $F$-value & $P$ value & Sum of squares & Mean square & $F$-value & $P$ value \\
\hline Model & 17876.21 & 1986.25 & 12.79 & $0.0014^{\mathrm{a}}$ & 1557.29 & 173.03 & 10.65 & $0.0025^{\mathrm{a}}$ \\
\hline$X_{1}$ & 724.99 & 724.99 & 4.67 & 0.0676 & 25.28 & 25.28 & 1.56 & 0.2524 \\
\hline$X_{2}$ & 567.05 & 567.05 & 3.65 & 0.0977 & 0.18 & 0.18 & 0.011 & 0.9191 \\
\hline$X_{3}$ & 4483.49 & 4483.49 & 28.86 & $0.0010^{\mathrm{a}}$ & 751.17 & 751.17 & 46.24 & $0.0003^{\mathrm{a}}$ \\
\hline$X_{1} X_{2}$ & 1190.35 & 1190.35 & 7.66 & $0.0278^{\mathrm{a}}$ & 240.72 & 240.72 & 14.82 & $0.0063^{\mathrm{a}}$ \\
\hline$X_{1} X_{3}$ & 607.99 & 607.99 & 3.91 & 0.0884 & 3.90 & 3.90 & 0.24 & 0.6391 \\
\hline$X_{2} X_{3}$ & 5.93 & 5.93 & 0.038 & 0.8506 & 0.21 & 0.21 & 0.013 & 0.9133 \\
\hline$X_{1}^{2}$ & 2304.09 & 2304.09 & 14.83 & $0.0063^{\mathrm{a}}$ & 117.79 & 117.79 & 7.25 & $0.0310^{\mathrm{a}}$ \\
\hline$X_{2}^{2}$ & 4077.02 & 4077.02 & 26.25 & $0.0014^{\mathrm{a}}$ & 2.86 & 2.86 & 0.18 & 0.6873 \\
\hline$X_{3}^{2}$ & 2850.96 & 2850.96 & 18.35 & $0.0036^{\mathrm{a}}$ & 382.74 & 382.74 & 23.56 & $0.0018^{\mathrm{a}}$ \\
\hline Residual & 1087.31 & 155.33 & & & 113.71 & 16.24 & & \\
\hline Lack of fit & 839.49 & 279.83 & 4.52 & 0.0897 & 62.06 & 20.69 & 1.60 & 0.3221 \\
\hline Cor total & 247.83 & 61.96 & & & 51.65 & 12.91 & & \\
\hline Pure error & 18963.53 & & & & 1671.0 & & & \\
\hline$R^{2}$ & 0.9427 & & & & 0.9320 & & & \\
\hline $\mathrm{CV} \%$ & $8.9 \%$ & & & & 5.28 & & & \\
\hline Adeq Precision & 9.208 & & & & 9.341 & & & \\
\hline
\end{tabular}

${ }^{a}$ Significant $(P<0.05)$. 


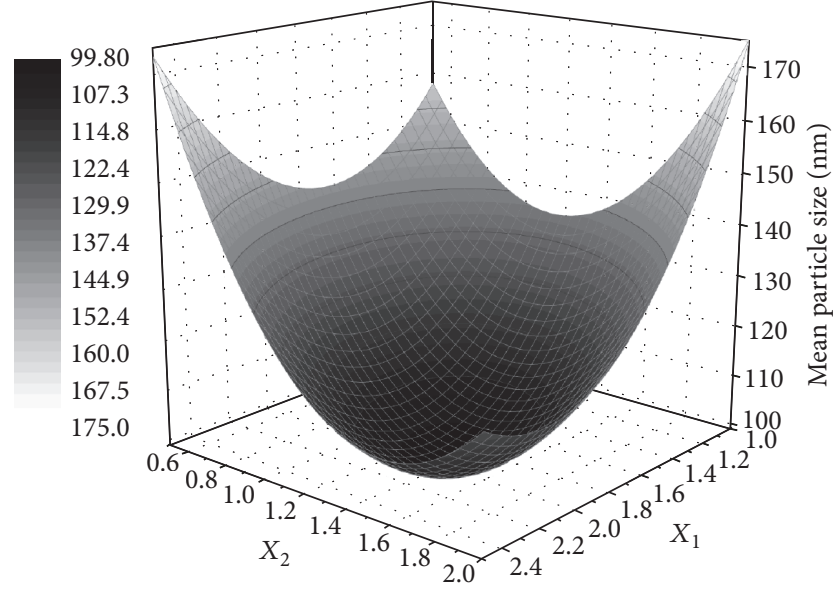

(a)

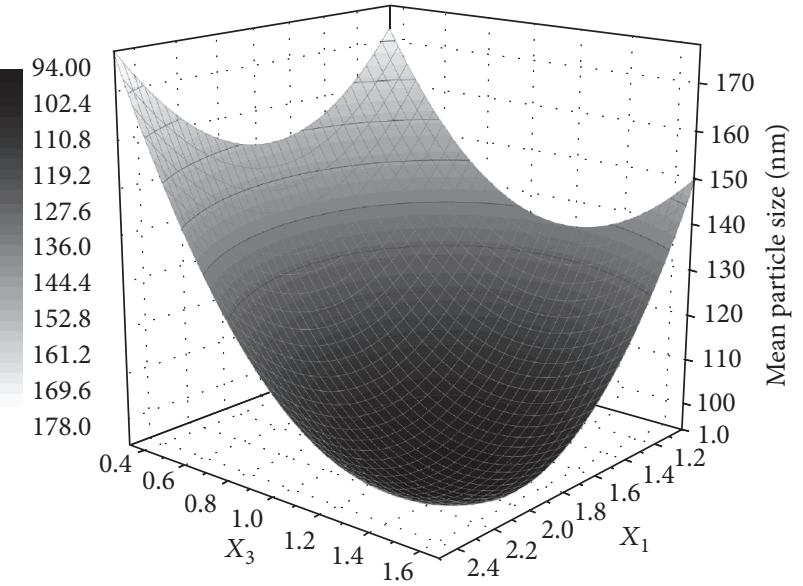

(b)

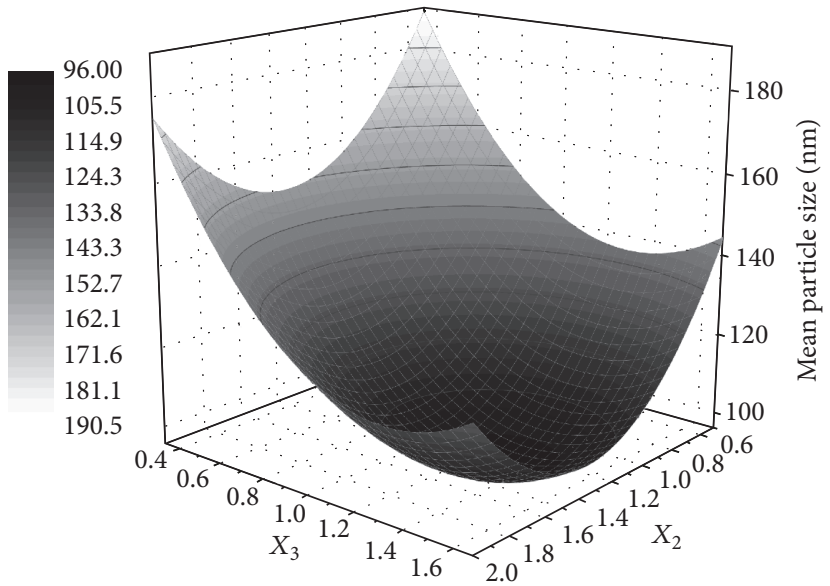

(c)

Figure 1: 3D response surface plot for the effect of emulsifier-to-lipid ratio $\left(X_{1}\right)$, lecithin-to Poloxamer 188 ratio $\left(X_{2}\right)$ and liquid lipid-to-solid liquid ratio $\left(X_{3}\right)$ on mean particle size of NOB-NLC. (a) Effect of $X_{1}-X_{2}$ interaction terms; (b) effect of $X_{1}-X_{3}$ interaction terms; (c) effect of $X_{2}-X_{3}$ interaction terms.

(Formulation (17)) with the selected levels of variables, whereas the average was found to be $76.30 \%$. The following quadratic equation showed the effect of various independent factors on EE:

$$
\begin{aligned}
Y_{2}= & 22.41+45.81 X_{1}+28.45 X_{2}+25.14 X_{3} \\
& -13.79 X_{1} X_{2}+1.97 X_{1} X_{3}-0.45 X_{2} X_{3} \\
& -9.40 X_{1}^{2}-1.47 X_{2}{ }^{2}-21.24 X_{3}{ }^{2} .
\end{aligned}
$$

The positive effect of variables $X_{1}, X_{2}$, and $X_{3}$ on EE could be seen in (4) as the positive value before these variables, as shown in the quadratic equation. Higher emulsifier-tolipid ratio could induce an increase in EE; the reason might be the availability of adequate emulsifier, which made the compound stay within the lipid particles and/or on the surface of the particles. Therefore, the liquid lipid-to-solid lipid ratio directly affects EE, which can be ascribed to the availability of higher amount of liquid lipid for compound entrapment. This can also be caused by the amphipathy of lecithin. Moreover, the interaction terms $X_{1}-X_{2}$ and $X_{2}-X_{3}$ exhibited a negative effect on particle size, whereas $X_{1}-X_{3}$ revealed positive effects on EE of NOB-NLC [34].

The $3 \mathrm{D}$ response surface graphs, which depict the interaction effects of $X_{1}-X_{2}, X_{1}-X_{3}$, and $X_{2}-X_{3}$ on EE, are presented in Figures 2(a)-2(c). The model $P$ value less than 0.05 and the $F$-value of 10.65 indicated the significance of the model for EE. Additionally, $P$ values of linear coefficient of $X_{3}$, interaction term coefficient of $X_{1} X_{2}$, and quadratic term coefficients of $X_{1}{ }^{2}$ and $X_{3}{ }^{2}$ were all above 0.05 , implying that they were significant on EE of NOB-NLC. Coefficient of correlation $\left(R^{2}=0.932\right)$ and the coefficient of variation were at a low value $(5.28 \%)$, which suggested a good relevance between predicted and experimental values. The model could be employed to navigate the design space demonstrating that the value of "Adeq Precision" is 9.341 [33].

3.2. Optimisation and Validation. The optimisation of NOBNLC preparation was chosen based on the prerequisite of fulfilling the minimum mean particle size and maximising the value of EE by applying the optimum formulation point 


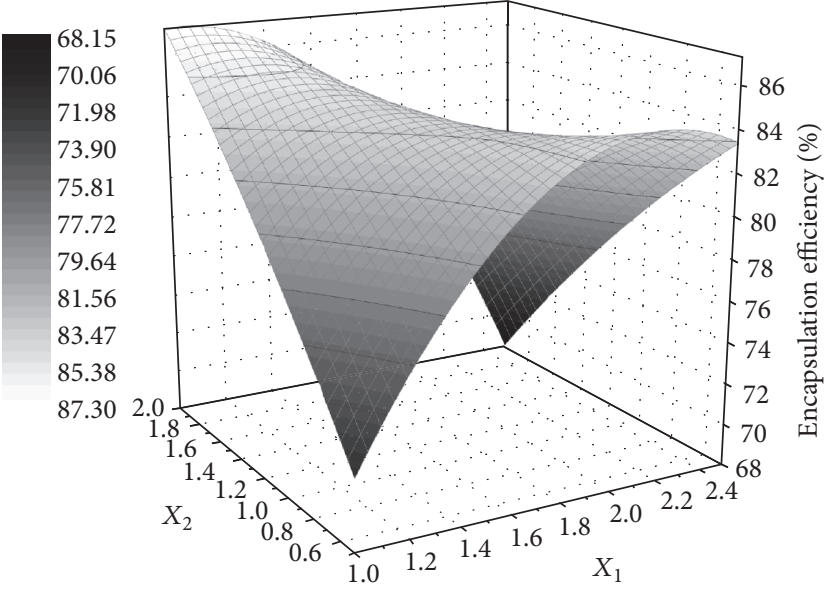

(a)

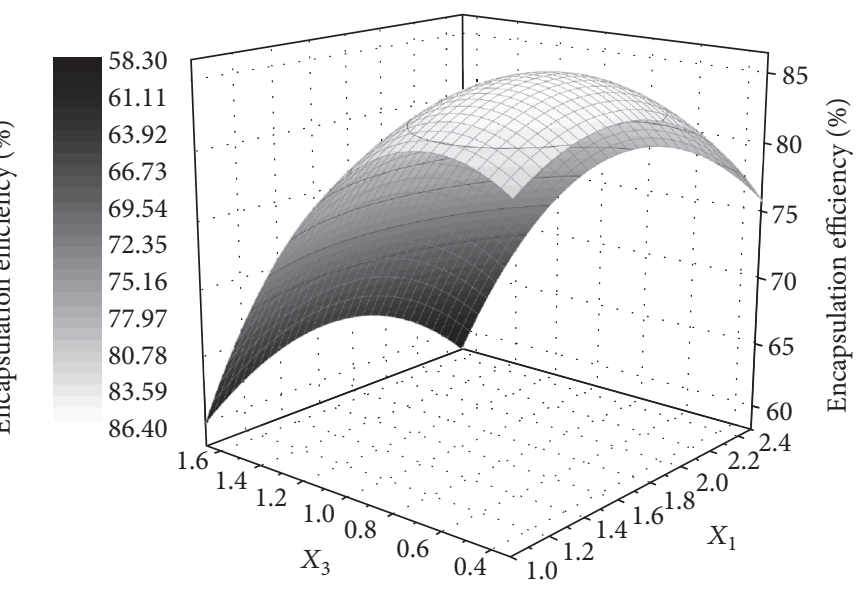

(b)

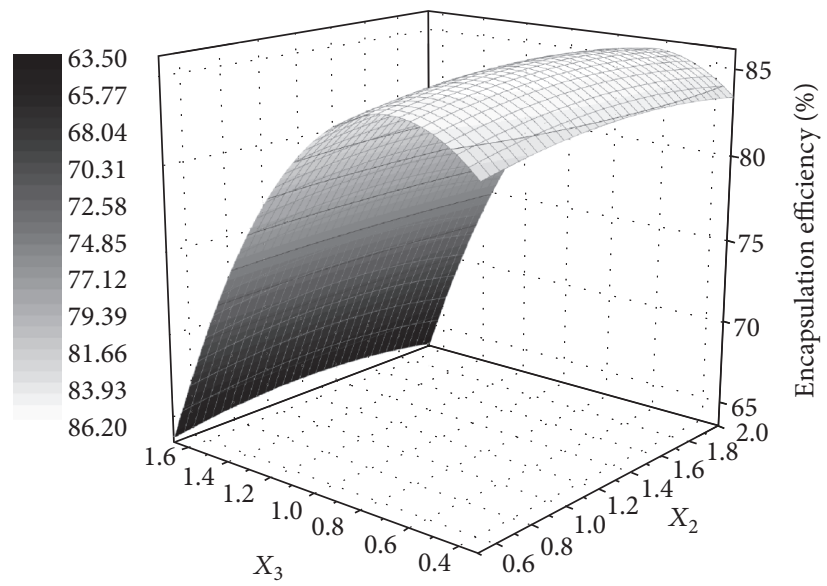

(c)

Figure 2: 3D response surface plot for the effect of emulsifier-to-lipid ratio $\left(X_{1}\right)$, lecithin-to Poloxamer 188 ratio $\left(X_{2}\right)$, and liquid lipid-tosolid liquid ratio $\left(X_{3}\right)$ on entrapment efficiency of NOB-NLC. (a) Effect of $X_{1}-X_{2}$ interaction terms; (b) effect of $X_{1}-X_{3}$ interaction terms; (c) effect of $X_{2}-X_{3}$ interaction terms.

of numerical method generated by Design Expert software. The optimised composition was selected as the emulsifierto-lipid ratio of 1.76 , lecithin-to-Poloxamer 188 ratio of 1.35 , and liquid lipid-to-solid lipid ratio of 0.95 , which were suggested to attain the prerequisites of the optimised NOBNLC formulation.

These observed responses of mean particle size (112.27 \pm $5.33 \mathrm{~nm})$ and EE (81.06 $\pm 6.02 \%)$, presented by the optimised NOB-NLC formulation, and the predicted value of mean particle size $(103.18 \mathrm{~nm})$ and $\mathrm{EE}(84.28 \%)$, generated by the Design Expert software, were found to be in good agreement. This finding demonstrates that the optimised formulation was reliable in predicting the response of the NOB-NLC system.

3.3. Surface Characterisation. The optimised NOB-NLC was homogeneous and slightly bluish, as shown in Figure 3(a). The particles exhibited a size of $112.27 \pm 5.33 \mathrm{~nm}$, with a PDI of $0.251 \pm 0.058$, an EE of $81.06 \pm 6.02 \%$, and zeta potential of $-35.1 \pm 2.94 \mathrm{mV}$. Studies of receiver biases suggest that the open endothelial gaps were in a range from $100 \mathrm{~nm}$ to $1000 \mathrm{~nm}$ [35], and particles of small size could trigger a long blood circulation as a free compound. The nanoparticles with a size of 100-300 $\mathrm{nm}$ have been found suitable to distribute in a tumor and easily transport through blood brain barriers [36]. PDI values below 0.3 indicated a narrow size distribution, excellent dispersity, and high homogeneity of nanoparticles [37]. The zeta potential played an important role in evaluating the stability of a nanoparticle system; high zeta potential can prevent particles from aggregating through electric repulsion. Currently, if the zeta potential is below $-30 \mathrm{mV}$ or above $+30 \mathrm{mV}$, the systems will be considered stable. Furthermore, the powerful statistical obstruction and beneficial static repulsion will contribute to additional stability [38, 39].

TEM images of NOB-NLC (Figure 3(b)) revealed that the optimised nanoparticles were well-identified as spherical, uniform, and nonadherent and were approximately $120 \mathrm{~nm}$ in diameter with a dense core (Figure 3(c)), which is accordant with the data estimated through DLS method [40]. Moreover, the particle size measured through DLS, called hydrodynamic 


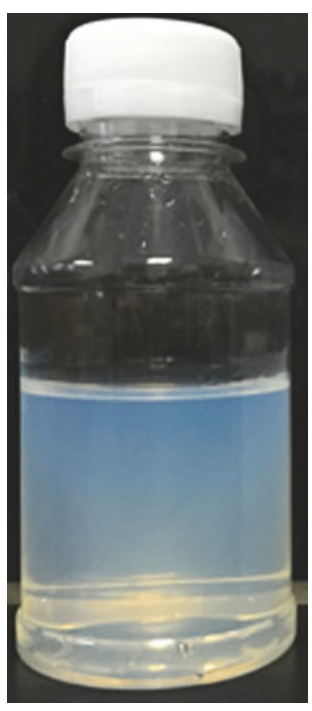

(a)

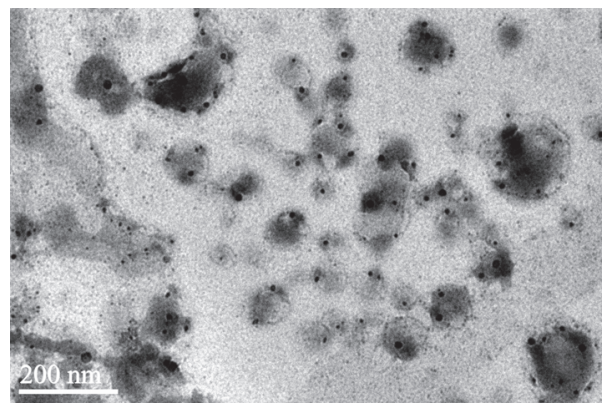

(b)

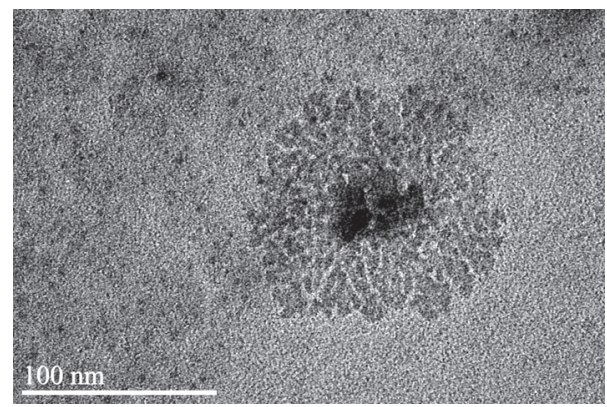

(c)

FIgURE 3: The extrinsic feature (a). TEM photomicrographs of NOB-NLC. Scale bar represents $200 \mathrm{~nm}$ (b) and $100 \mathrm{~nm}$ (c).

size, was found to be larger than that of the TEM results, called actual size. These differences are hypothesised to be attributed to different size measurement methods, as the solvent layer attached to the particle is measured in DLS [36]. Different morphologies have been reported in much of the research in NLC; nevertheless, spherical morphology that has been discovered holds a greater proportion [12]. Previous study indicated that the differences of compositions of lipid and surfactants or photographic equipment employed may result in the distinction in morphology of NLC [41].

3.4. Solid-State Characterisation of NOB-NLC Systems: DSC Data. Figure 6 shows the modulated DSC scans of the freezedried NOB-NLC, blank NLC, and NOB. A sharp endothermic peak was observed in the crystalline $\mathrm{NOB}$ at $137.03^{\circ} \mathrm{C}$, which was in concert with its melting point (Figure 4(c)). The melting of blank NLC peaked at $48.73^{\circ} \mathrm{C}$ with a small shape (Figure 4(a)), and a lower melting peak of NOB-NLC appeared at $50.58^{\circ} \mathrm{C}$ in the thermogram (Figure $4(\mathrm{~b})$ ). In the NOB-NLC formulations, the NOB melting could not be detected, which could be attributed to the existence of NOB in the amorphous state or in a molecularly dispersed state within the lipid matrix. Even if a small fraction of NOB is present in NOB-NLC as undissolved material in the crystalline state, the relatively low compound loading of $\mathrm{NOB}$ in NOB-NLC would render NOB-NLC improbable to detect the melting event of any such fraction using a DSC-based technique [42]. This phenomenon can also be ascribed to the interference of NOB and the surfactants in NOB-NLC crystallisation [43]. Furthermore, according to Gibbs-Thomson equation, particle size also has a conspicuous effect on the melting endotherm of lipid particles in nanoscale range [44]. Different lipid nanoparticles melted at different temperatures and led to peak broadening and a change in melting transition to lower temperature on the basis of this equation, as was different from that of mass lipid [36].

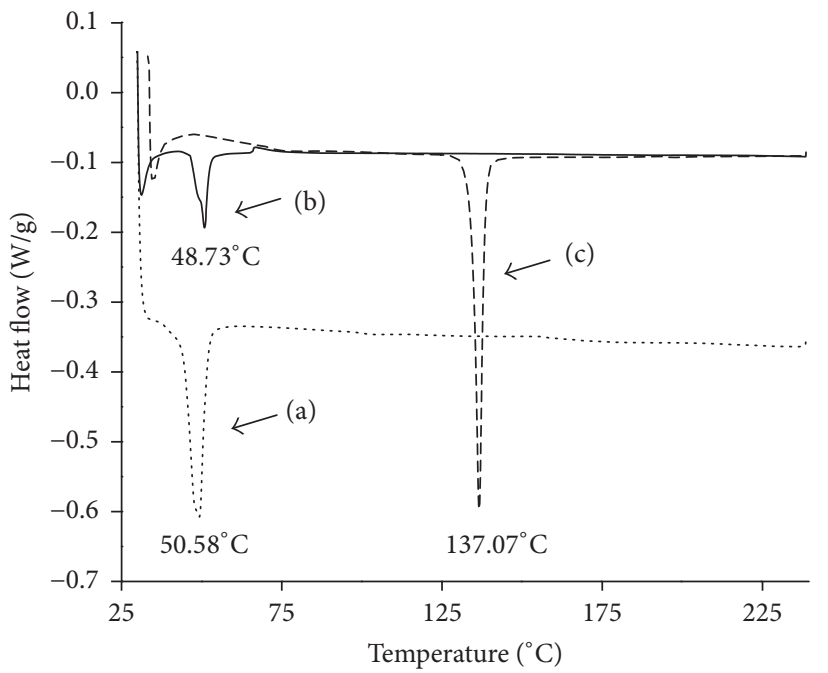

FIGURE 4: DSC analysis of blank NLC (a), NOB-NLC (b), and NOB (c).

3.5. Solid-State Characterisation of NOB-NLC Systems: FT-IR Data. The overlay spectra of freeze-dried NOB-NLC, blank NLC, and NOB are shown in Figure 6. The IR spectrum of NOB (Figure 6(c)) revealed the characteristic absorption bands at $1643.54(-\mathrm{C}=\mathrm{O}$, aromatic ketone), 1587.13 (C-C, aromatic ring), 1013.89 (C-O stretch), and $2944.77 \mathrm{~cm}^{-1}$ (C$\mathrm{H}$ stretch). In NOB-NLC, these peaks appeared at 1644.02 ($\mathrm{C}=\mathrm{O}$, aromatic ketone), 1586.16 (C-C, aromatic ring), 1014.86 (C-O stretch), and $2952.48 \mathrm{~cm}^{-1}$ (C-H stretch) (Figure 5(c)). This confirmed the presence of NOB in NOB-NLC, indicating that no chemical interaction between the compound and lipid excipients took place. The IR spectra of blank NLC (Figure 5(a)) and NOB-NLC (Figure 5(b)) were clearly suggestive of the presence of another crystalline form in 


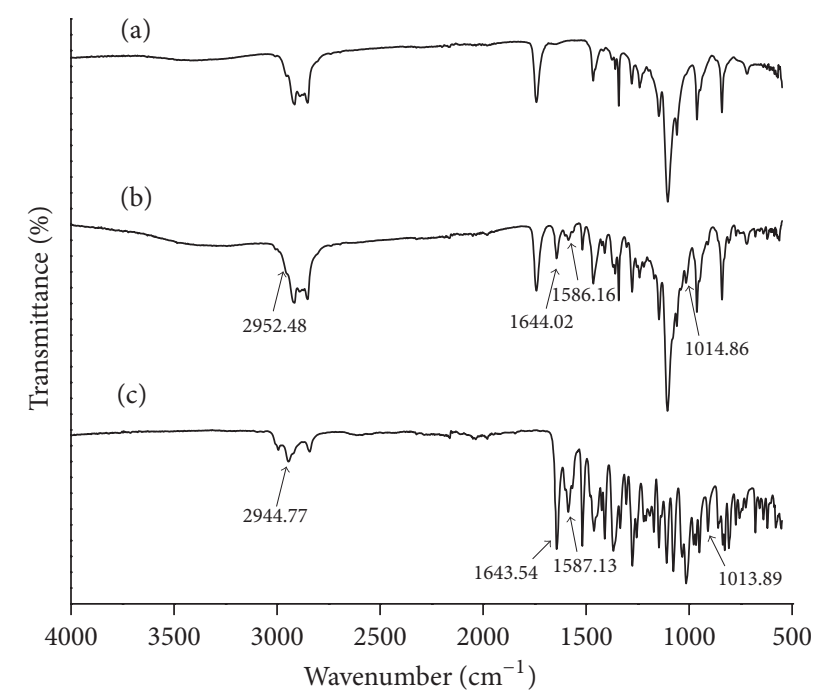

FIGURE 5: FT-IR spectra of blank NLC (a), NOB-NLC (b), and NOB (c).

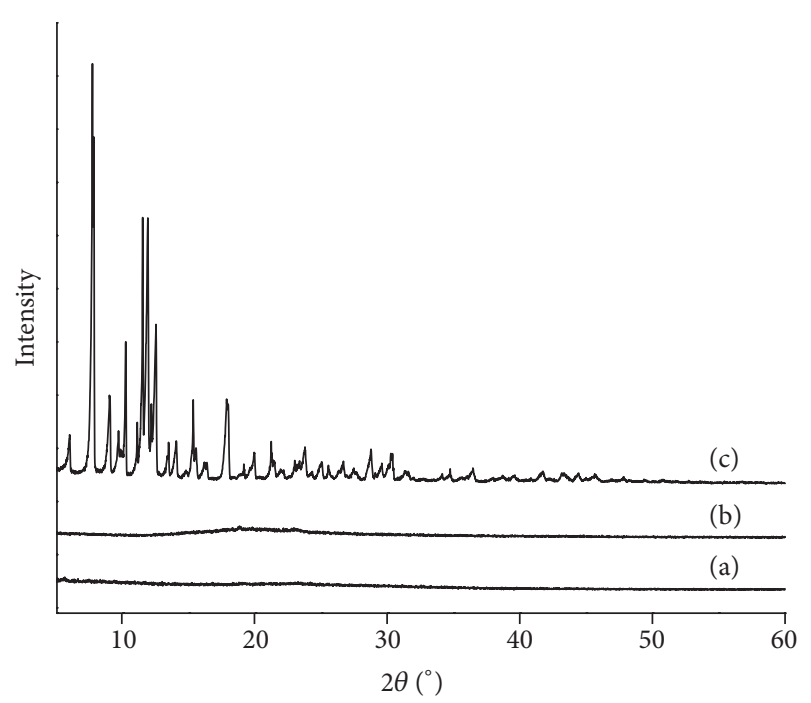

Figure 6: X-ray diffractograms of blank NLC (a), NOB-NLC (b), and $\operatorname{NOB}(\mathrm{c})$.

NOB-NLC, which was further confirmed using DSC. The slight shift and decrease of absorption bands of NOB in NOB-NLC can be ascribed to a transformation in molecular environment and intermolecular interactions accompanied by dispersed compound molecule in lipid matrix [36].

3.6. Solid-State Characterisation of NOB-NLC Systems: XRD Data. XRD experiments were performed to investigate the changes of the microstructure in the lipid crystallisation process (Figure 6). The XRD spectra of NOB in Figure 6(c) showed distinct and intense peaks at $2 \theta$ scale, indicating the high crystalline structure of NOB (i.e., three sharp peaks at $2 \theta=7.68^{\circ}, 11.47^{\circ}$, and $11.86^{\circ}$; five medium-intensity peaks at $2 \theta=8.99^{\circ}, 10.19^{\circ}, 12.46^{\circ}, 15.28^{\circ}$, and $18.84^{\circ}$; and several lowintensity peaks at $2 \theta=5.97^{\circ}, 9.64^{\circ}$, and $13.42^{\circ}$ ). Characteristic peaks of NOB were absent from the XRD pattern of NOBNLC (Figure 6(b)), indicating that the NOB molecule lost its crystalline nature and formed an amorphous complex with the NLC matrix. This discovery corresponds to the result from the DSC analysis, providing evidence of the phase transition of NOB from crystalline to amorphous during processing [45].

\section{Conclusion}

NOB-loaded NLCs were successfully developed through a high-pressure homogenisation technique and optimised by seventeen-run, three-factor, three-level BBD. The lecithin-toPoloxamer 188 ratio and the liquid lipid-to-solid lipid ratio were crucial determinants for NOB-NLC production as these factors influenced the physical properties of NOB-NLC. The optimised NOB-NLC manifested a size of $112.27 \pm 5.33 \mathrm{~nm}$ with a polydispersity of $0.251 \pm 0.058$, an EE of $81.06 \pm 6.02 \%$, and zeta potential of $-35.1 \pm 2.94 \mathrm{mV}$. Particle size and TEM study confirmed nanosized discrete spherical globules with a smooth surface area. DSC and XRD confirmed the transformation of the crystalline nature of NOB into an amorphous nature, which played an important role in the enhancement of absorption rate, followed by NOB bioavailability. FT-IR analysis confirmed the efficient entrapment of NOB in NOBNLCs. Thus, this present research demonstrated that the optimised formulation has potential in delivering NOB and other lipophilic compounds. Future research should focus on the appraisal of the practical potential of the optimum formulation in food-relevant models and actual food systems during production and storage.

\section{Conflicts of Interest}

The authors declare that they have no conflicts of interest.

\section{Acknowledgments}

This research is funded by Chongqing Research Program of Basic Research and Frontier Technology (no. cstc2016jcyjA0552), Special Fund for Agro-Scientific Research in the Public Interest (201303076-07), and Chinese National Natural Science Foundation (31601396).

\section{References}

[1] S. Li, H. Wang, L. Guo, H. Zhao, and C.-T. Ho, "Chemistry and bioactivity of nobiletin and its metabolites," Journal of Functional Foods, vol. 6, no. 1, pp. 2-10, 2014.

[2] N. Sp, D. Kang, Y. Joung et al., "Nobiletin inhibits angiogenesis by regulating Src/FAK/STAT3-mediated signaling through PXN in ER+ breast cancer cells," International Journal of Molecular Sciences, vol. 18, no. 5, p. 935, 2017.

[3] Y. Wu, W. Zhang, M. Li, D. Cao, X. Yang, and J. Gong, "Nobiletin ameliorates ischemia-reperfusion injury by suppressing the function of Kupffer cells after liver transplantation in rats," Biomedicine Pharmacotherapy, vol. 89, pp. 732-741, 2017.

[4] Y. Zheng, J. Bu, L. Yu, J. Chen, and H. Liu, "Nobiletin improves propofol-induced neuroprotection via regulating Akt/mTOR 
and TLR 4/NF- $\kappa \mathrm{B}$ signaling in ischemic brain injury in rats," Biomedicine Pharmacotherapy, vol. 91, pp. 494-503, 2017.

[5] N. Braidy, S. Behzad, S. Habtemariam et al., "Neuroprotective effects of citrus fruit-derived flavonoids, nobiletin and tangeretin in alzheimer's and parkinson's disease," CNS and Neurological Disorders Drug Targets, 2017.

[6] S. Onoue, A. Uchida, H. Takahashi et al., "Development of highenergy amorphous solid dispersion of nanosized nobiletin, a citrus polymethoxylated flavone, with improved oral bioavailability," Journal of Pharmaceutical Sciences, vol. 100, no. 9, pp. 3793-3801, 2011.

[7] C. M. O’Driscoll and B. T. Griffin, "Biopharmaceutical challenges associated with drugs with low aqueous solubility-The potential impact of lipid-based formulations," Advanced Drug Delivery Reviews, vol. 60, no. 6, pp. 617-624, 2008.

[8] R. H. Müller, M. Radtke, and S. A. Wissing, "Solid lipid nanoparticles (SLN) and nanostructured lipid carriers (NLC) in cosmetic and dermatological preparations," Advanced Drug Delivery Reviews, no. 54, pp. 131-155, 2002.

[9] R. H. Müller, M. Radtke, and S. A. Wissing, "Nanostructured lipid matrices for improved microencapsulation of drugs," International Journal of Pharmaceutics, vol. 242, no. 1-2, pp. 121$128,2002$.

[10] M. D. Joshi and R. H. Müller, "Lipid nanoparticles for parenteral delivery of actives," European Journal of Pharmaceutics and Biopharmaceutics, vol. 71, no. 2, pp. 161-172, 2009.

[11] R. H. Müller, R. D. Petersen, A. Hommoss, and J. Pardeike, "Nanostructured lipid carriers (NLC) in cosmetic dermal products," Advanced Drug Delivery Reviews, vol. 59, no. 6, pp. 522530, 2007.

[12] C. L. Fang, S. A. Al-Suwayeh, and J. Y. Fang, "Nanostructured lipid carriers (NLCs) for drug delivery and targeting," Recent Patents on Nanotechnology, vol. 7, no. 1, pp. 41-55, 2013.

[13] F. Tamjidi, M. Shahedi, J. Varshosaz, and A. Nasirpour, "Nanostructured lipid carriers (NLC): a potential delivery system for bioactive food molecules," Innovative Food Science and Emerging Technologies, vol. 19, pp. 29-43, 2013.

[14] J. Yao, J. P. Zhou, Q. N. Ping, Y. Lu, and L. Chen, “Distribution of nobiletin chitosan-based microemulsions in brain following i.v. injection in mice," International Journal of Pharmaceutics, vol. 352, no. 1-2, pp. 256-262, 2008.

[15] H. Chen, Y. An, X. Yan, D. J. McClements, B. Li, and Y. Li, "Designing self-nanoemulsifying delivery systems to enhance bioaccessibility of hydrophobic bioactives (nobiletin): influence ofhydroxypropyl methylcellulose and thermal processing," Food Hydrocolloids, vol. 51, pp. 395-404, 2015.

[16] A. G. Luque-Alcaraz, J. Lizardi, F. M. Goycoolea et al., "Characterization and antiproliferative activity of nobiletin-loaded chitosan nanoparticles," Journal of Nanomaterials, vol. 2012, Article ID 265161, 7 pages, 2012.

[17] W. Lin, J. Yao, and J. Zhou, "Preparation of self-assemble nobiletin proliposomes and its pharmacokinetics in rats," Acta Pharmaceutica Sinica, vol. 44, no. 02, pp. 192-196, 2009.

[18] X. F. Zhang, C. F. Xing, and Q. T. Guo, "Preparation of nobiletin solid lipid nanoparticles," Chinese Traditional Patent Medicine, vol. 36, no. 09, pp. 1857-1862, 2014.

[19] L. Lei, Y. Zhang, L. He, S. Wu, B. Li, and Y. Li, "Fabrication of nanoemulsion-filled alginate hydrogel to control the digestion behavior of hydrophobic nobiletin," LWT-Food Science and Technology, vol. 82, pp. 260-267, 2017.
[20] G. Calixto, J. Bernegossi, B. Fonseca-Santos, and M. Chorilli, "Nanotechnology-based drug delivery systems for treatment of oral cancer: a review," International Journal of Nanomedicine, vol. 9, no. 1, pp. 3719-3735, 2014.

[21] M. Li, Y. Zheng, F.-Y. Shan, J. Zhou, T. Gong, and Z.-R. Zhang, "Development of ionic-complex-based nanostructured lipid carriers to improve the pharmacokinetic profiles of breviscapine," Acta Pharmacologica Sinica, vol. 34, no. 8, pp. 1108-1115, 2013.

[22] M. Kamran, A. Ahad, M. Aqil, S. S. Imam, Y. Sultana, and A. Ali, "Design, formulation and optimization of novel soft nanocarriers for transdermal olmesartan medoxomil delivery: in vitro characterization and in vivo pharmacokinetic assessment," International Journal of Pharmaceutics, vol. 505, no. 1-2, pp. 147158,2016

[23] C. L. Ngan, M. Basri, F. F. Lye et al., "Comparison of BoxBehnken and central composite designs in optimization of fullerene loaded palm-based nano-emulsions for cosmeceutical application," Industrial Crops and Products, vol. 59, pp. 309-317, 2014.

[24] M. Shangguan, Y. Lu, J. Qi et al., "Binary lipids-based nanostructured lipid carriers for improved oral bioavailability of silymarin," Journal of Biomaterials Applications, vol. 28, no. 6, pp. 887-896, 2014.

[25] M. Sun, S. Nie, X. Pan, R. Zhang, Z. Fan, and S. Wang, "Quercetin-nanostructured lipid carriers: characteristics and antibreast cancer activities in vitro," Colloids and Surfaces B: Biointerfaces, vol. 113, pp. 15-24, 2014.

[26] E. Esposito, L. Ravani, M. Drechsler et al., "Cannabinoid antagonist in nanostructured lipid carriers (NLCs): design, characterization and in vivo study," Materials Science and Engineering C, vol. 48, pp. 328-336, 2015.

[27] G. Fang, B. Tang, Y. Chao et al., "Cysteine-functionalized nanostructured lipid carriers for oral delivery of docetaxel: a permeability and pharmacokinetic study," Molecular Pharmaceutics, vol. 12, no. 7, pp. 2384-2395, 2015.

[28] R. Qi, Y.-Z. Li, C. Chen et al., "G5-PEG PAMAM dendrimer incorporating nanostructured lipid carriers enhance oral bioavailability and plasma lipid-lowering effect of probucol," Journal of Controlled Release, vol. 210, pp. 160-168, 2015.

[29] T.-H. Wu, F.-L. Yen, L.-T. Lin, T.-R. Tsai, C.-C. Lin, and T.M. Cham, "Preparation, physicochemical characterization, and antioxidant effects of quercetin nanoparticles," International Journal of Pharmaceutics, vol. 346, no. 1-2, pp. 160-168, 2008.

[30] H. Li, X. Zhao, Y. Ma, G. Zhai, L. Li, and H. Lou, "Enhancement of gastrointestinal absorption of quercetin by solid lipid nanoparticles," Journal of Controlled Release, vol. 133, no. 3, pp. 238244, 2009.

[31] J. Das Neves and B. Sarmento, "Precise engineering of dapivirine-loaded nanoparticles for the development of antiHIV vaginal microbicides," Acta Biomaterialia, vol. 18, pp. 7787, 2015.

[32] H. Rostami and S. M. T. Gharibzahedi, "Microwave-assisted extraction of jujube polysaccharide: optimization, purification and functional characterization," Carbohydrate Polymers, vol. 143, pp. 100-107, 2016.

[33] Z. Ye, W. Wang, Q. Yuan et al., "Box-Behnken design for extraction optimization, characterization and in vitro antioxidant activity of Cicer arietinum L. hull polysaccharides," Carbohydrate Polymers, vol. 147, pp. 354-364, 2016.

[34] M. Pradhan, D. Singh, S. N. Murthy, and M. R. Singh, "Design, characterization and skin permeating potential of Fluocinolone 
acetonide loaded nanostructured lipid carriers for topical treatment of psoriasis," Steroids, vol. 101, article no. 7791, pp. 56-63, 2015.

[35] S. K. Hobbs, W. L. Monsky, F. Yuan et al., "Regulation of transport pathways in tumor vessels: role of tumor type and microenvironment," Proceedings of the National Academy of Sciences of the United States of America, vol. 95, no. 8, pp. 4607-4612, 1998.

[36] P. Dadhania, P. R. Vuddanda, A. Jain, S. Velaga, and S. Singh, "Intranasal delivery of asenapine loaded nanostructured lipid carriers: formulation, characterization, pharmacokinetic and behavioural assessment," RSC Advances, vol. 6, no. 3, pp. 20322045, 2016.

[37] J. Li, J. Wu, J. Zhang, Y. Wang, L. Fang, and Q. Shen, "Oral bioavailability and evaluation of docetaxel-nicotinamide complex loaded chitosan nanoparticles," RSC Advances, vol. 6, no. 42, pp. 35354-35364, 2016.

[38] J.-H. Park, S.-J. Ban, T. Ahmed et al., "Development of DHI-180-3 loaded lipid nanoparticle for photodynamic therapy," International Journal of Pharmaceutics, vol. 491, no. 1-2, pp. 393401, 2015.

[39] H. Chen, Y. Wang, Y. Zhai, G. Zhai, Z. Wang, and J. Liu, "Development of a ropivacaine-loaded nanostructured lipid carrier formulation for transdermal delivery," Colloids and Surfaces A: Physicochemical and Engineering Aspects, vol. 465, pp. 130-136, 2015.

[40] B. Gupta, B. K. Poudel, T. H. Tran et al., "Modulation of pharmacokinetic and cytotoxicity profile of imatinib base by employing optimized nanostructured lipid carriers," Pharmaceutical Research, vol. 32, no. 9, article no. 1673, pp. 2912-2927, 2015.

[41] C. Liu and C. Wu, "Optimization of nanostructured lipid carriers for lutein delivery," Colloids and Surfaces A: Physicochemical and Engineering Aspects, vol. 353, no. 2-3, pp. 149-156, 2010.

[42] S. Bose and B. Michniak-Kohn, "Preparation and characterization of lipid based nanosystems for topical delivery of quercetin," European Journal of Pharmaceutical Sciences, vol. 48, no. 3, pp. 442-452, 2013.

[43] F. Tamjidi, M. Shahedi, J. Varshosaz, and A. Nasirpour, "Design and characterization of astaxanthin-loaded nanostructured lipid carriers," Innovative Food Science and Emerging Technologies, vol. 26, pp. 366-374, 2014.

[44] H. Bunjes and T. Unruh, "Characterization of lipid nanoparticles by differential scanning calorimetry, X-ray and neutron scattering," Advanced Drug Delivery Reviews, vol. 59, no. 6, pp. 379-402, 2007.

[45] T. H. I. Tran, T. Ramasamy, D. H. I. Truong, H.-G. Choi, C. S. O. Yong, and J. O. H. Kim, "Preparation and characterization of fenofibrate-loaded nanostructured lipid carriers for oral bioavailability enhancement," AAPS PharmSciTech, vol. 15, no. 6, pp. 1509-1515, 2014. 

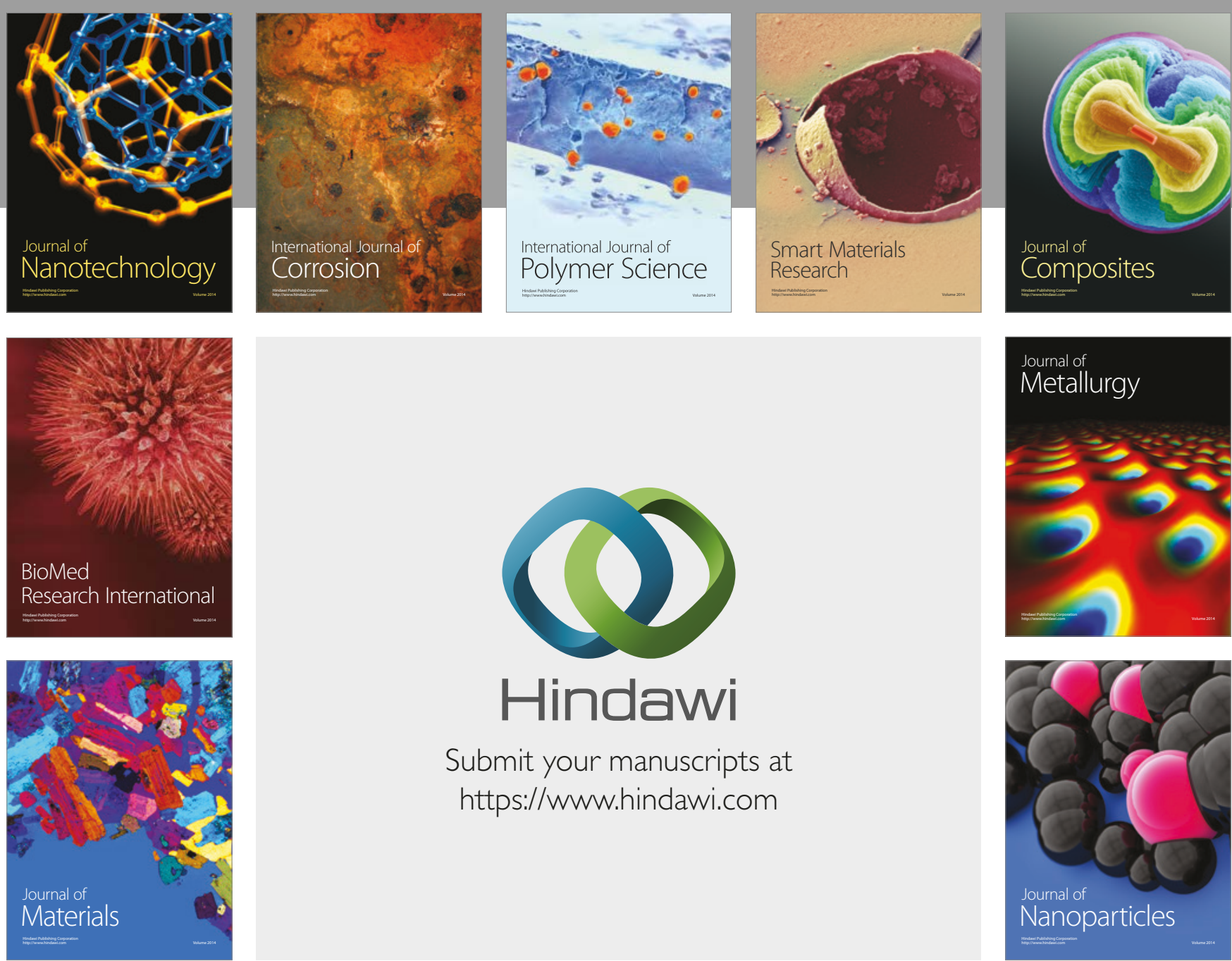

\section{Hindawi}

Submit your manuscripts at

https://www.hindawi.com
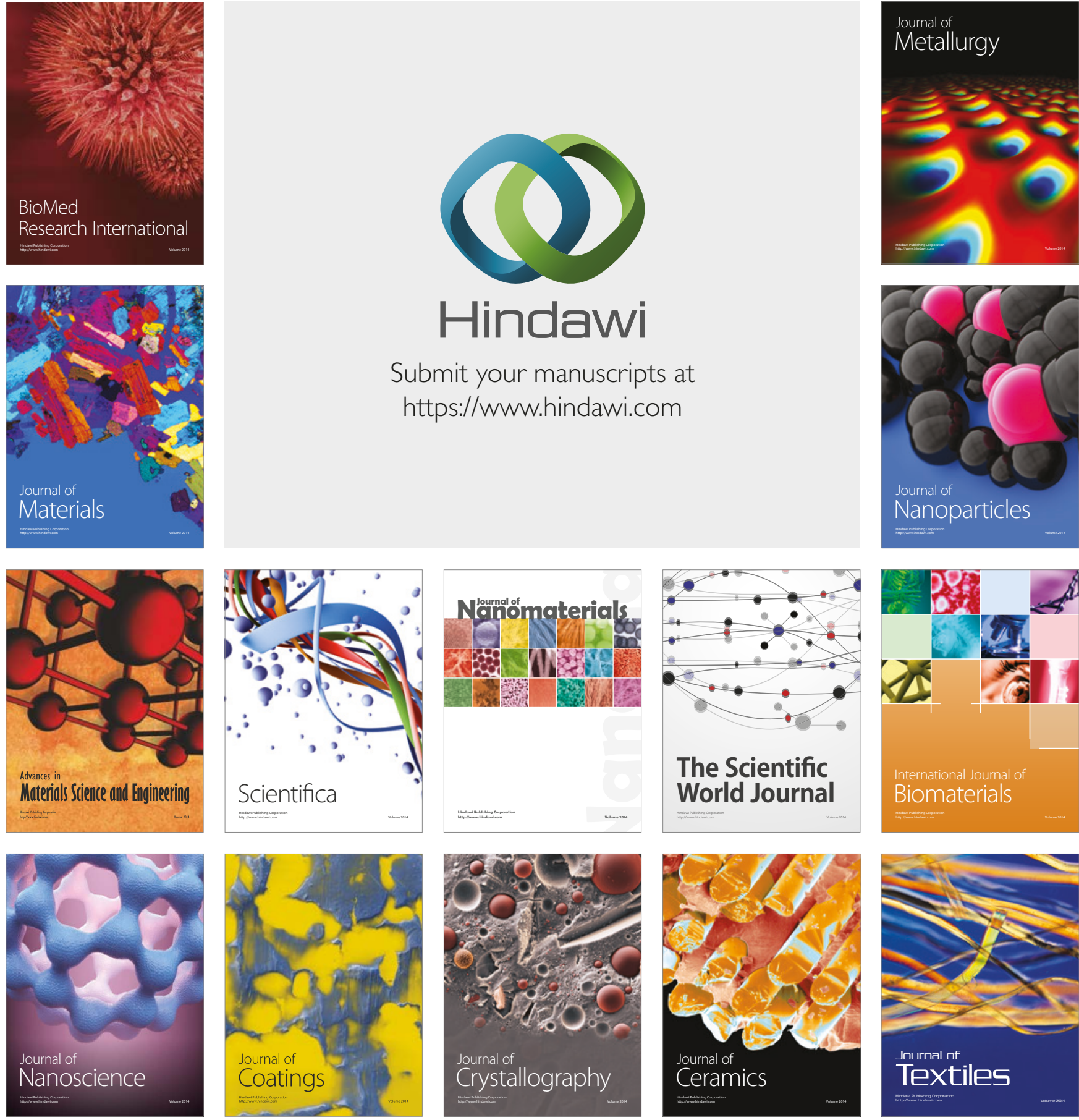

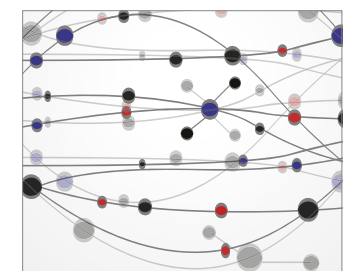

The Scientific World Journal
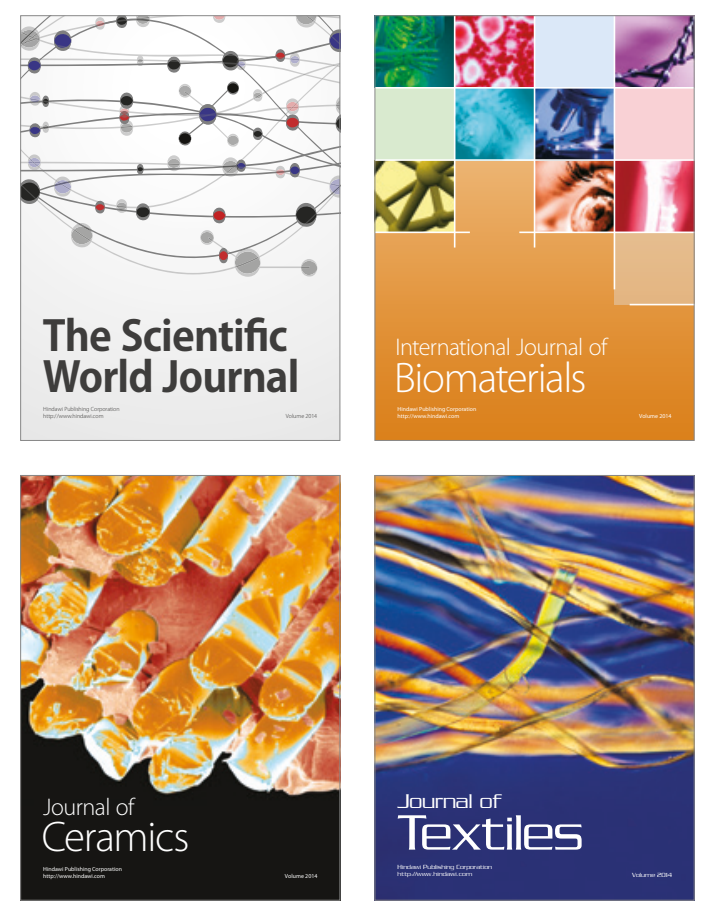